\title{
ARCHAEOLOGICAL SITE MONITORING: UAV PHOTOGRAMMETRY CAN BE AN ANSWER
}

\author{
F. Rinaudo ${ }^{\text {a, }}$, F. Chiabrando ${ }^{\text {a }}$, A. Lingua ${ }^{\text {b }}$, A. Spanò ${ }^{\text {a }}$ \\ ${ }^{\text {a }}$ DAD - Politecnico di Torino, Viale Mattioli 39, 10125 Torino \\ ${ }^{\mathrm{b}}$ DIATI - Politecnico di Torino, Corso Duca degli Abruzzi, 24, 10129 Torino \\ (fulvio.rinaudo, filiberto.chiabrando, antonia.spano, andrea.lingua)@polito.it
}

Commission V, WG V/2

KEY WORDS: Archaeology, Monitoring, Instruments, Aerial, Performance

\begin{abstract}
:
During archaeological excavations it is important to monitor the new excavated areas and findings day by day in order to be able to plan future excavation activities. At present, this daily activity is usually performed by using total stations, which survey the changes of the archaeological site: the surveyors are asked to produce day by day draft plans and sections which allow archaeologists to plan their future activities. The survey is realized during the excavations or just at the end of every working day and drawings have to be produced as soon as possible in order to allow the comprehension of the work done and to plan the activities for the following day. By using this technique, all the measurements, even those not necessary for the day after, have to be acquired in order to avoid a 'loss of memory'.

A possible alternative to this traditional approach is aerial photogrammetry, if the images can be acquired quickly and at a taken distance able to guarantee the necessary accuracy of a few centimeters.

Today the use of UAVs (Unmanned Aerial Vehicles) can be considered a proven technology able to acquire images at distances ranging from $4 \mathrm{~m}$ up to $20 \mathrm{~m}$ : and therefore as a possible monitoring system to provide the necessary information to the archaeologists day by day. The control network, usually present at each archaeological site, can give the stable control points useful for orienting a photogrammetric block acquired by using an UAV equipped with a calibrated digital camera and a navigation control system able to drive the aircraft following a pre-planned flight scheme. Modern digital photogrammetric software can solve for the block orientation and generate a DSM automatically, allowing rapid orthophoto generation and the possibility of producing sections and plans.

The present paper describes a low cost UAV system realized by the research group of the Politecnico di Torino and tested on a Roman villa archaeological site located in Aquileia (Italy), a well-known UNESCO WHL site. The results of automatic orientation and orthophoto production are described in terms of their accuracy and the completeness of information guaranteed for archaeological site excavation management.
\end{abstract}

\section{INTRODUCTION}

The metric documentation of archaeological sites has by now reached a high degree of definition and increasingly pushes the actors involved in the process of documentation and metric survey to the use of new methodologies. There has taken place a gradual but continuous transition from traditional methods of data collection, such as the direct survey system, to those involving a higher degree of automation, such as laser scanners and digital photogrammetry, and including the use of unconventional systems, such as UAVs (Unmanned Aerial Vehicles), RPVs (Remote Piloted Vehicles), or non-motorized platforms including aerostatic balloons [Altan, 2004; Celykoian, 2003; Fotinopoulos, 2004], kites [Aber, 2002; Bitelli, 2003; Bogacki, 2008], or telescopic arms [Chiabrando, 2010].

The common aim of these systems is to obtain aerial images at very low altitude useful both as pure documentation of the excavation [Verhoeven, 2009] and as the primary data which, thanks to the use of methodologies and techniques typical of Geomatics, can be transformed into the metrically reliable products needed for a correct representation and knowledge of the investigated area.

The possibilities offered by digital photogrammetry and the continuous development of compact ultra-high resolution digital cameras, associated with a greater and greater miniaturization and sustainability in terms of cost of those sensors, has enabled, in recent years, a substantial increase in the number of UAV systems used for the creation of photogrammetric measurements in the fields of archeology, architecture, and environmental metric documentation.

The development of these platforms started in the 50 s for military purposes; at present, these systems have evolved with levels of technology of ever-higher performance.

Today, UAVs are used both for military and civil applications: for reconnaissance, surveillance, atmospheric measurements, assessment of damages, and the mapping of natural hazards and other phenomena for which it is not possible to have a direct access to the site.

These aircraft are classified on the basis of different specifications: the more robust classification was drafted by the Unmanned Vehicle Systems International Association [International Unmanned Aerial System Community, 2008]. According to this classification, UAVs are divided into three main categories according to their possible use: Tactical, Strategic, and Special Purpose. These three main categories are divided into subcategories on the basis of their characteristics 
and performance: the capability of flight, maximum reachable flight height, weight, and autonomy. The systems that are most used today for metric surveys or documentation at a very large scale can be regarded as belonging to the Tactical category (with subcategories micro and mini UAVs). Table 1 gives the characteristics of the systems that relate to the category called Tactical.

The different experiments performed at the national and international level are about the use of different approaches with different platforms; from non-motorized systems, already mentioned (balloons, kites, etc.), to those that involve the use of electric or combustion-based engines such as: fixed-wing aircraft [Bendea, 2008; Cunningham, 2011; Haala, 2011; Scholtz, 2011] and mini-helicopters [Eisenbeiss, 2005; Patias , 2007, 2009; Remondino, 2009, 2011; Skarlatos, 2004; Spatalas, 2006; Theodoridou, 2000; Tokmakidis, 2000; Scaioni, 2009].

Today, as a result of the experience and testing performed on various platforms [Chiabrando, 2011], the research group of the Politecnico di Torino is now oriented to the use of minihelicopters which are not single-helix but are instead supported by systems with four, six, and eight propellers (the so-called hexa or hepta-copter), with: better handling and stability in flight phase compared to traditional mini-helicopters with a single helix; reduced costs by considering the increasing demand of these platforms; a simplification in control operations performed by the operator devoted to the phase of flight and image acquisition.

\begin{tabular}{|l|l|l|l|l|}
\hline $\begin{array}{l}\text { Tactical UAVs } \\
\text { Sub-categories }\end{array}$ & Acronym & $\begin{array}{l}\text { Max high } \\
{[\mathrm{m}]}\end{array}$ & $\begin{array}{l}\text { Autonomy } \\
{[\text { hours }]}\end{array}$ & $\begin{array}{l}\text { Weight } \\
{[\mathrm{Kg}]}\end{array}$ \\
\hline Micro & $\begin{array}{l}\mu \\
(\text { Micro })\end{array}$ & 250 & 1 & $<5$ \\
\hline Mini & Mini & $150-300$ & $<2$ & 150 \\
\hline Close Range & CR & 3000 & $2-4$ & 150 \\
\hline Short Range & SR & 3000 & $3-6$ & 200 \\
\hline $\begin{array}{l}\text { Medium } \\
\text { Range }\end{array}$ & MR & 5000 & $6-10$ & 1250 \\
\hline $\begin{array}{l}\text { Medium } \\
\text { Range } \\
\text { Endurance }\end{array}$ & MRE & 8000 & $10-18$ & 1250 \\
\hline $\begin{array}{l}\text { Low Altitude } \\
\text { Deep } \\
\text { Penetration }\end{array}$ & LADP & $50-9000$ & $0,5-1$ & 350 \\
\hline $\begin{array}{l}\text { Low Altitude } \\
\text { Long } \\
\text { Endurance }\end{array}$ & LALE & 3000 & $>24$ & $<30$ \\
\hline $\begin{array}{l}\text { Medium } \\
\text { Altitude Long } \\
\text { Endurance }\end{array}$ & MALE & 14000 & $24-48$ & 1500 \\
\hline
\end{tabular}

Table 1. Classification of Tactical UAVs by the Unmanned Vehicle Systems International Association

The work presented in the present article describes the first experiments performed with a hexa-copter developed by the research group of the Politecnico di Torino. In particular, it will describe the characteristics of the platform, the management of the acquisition stage, the results obtained after an aerial triangulation adjustment, the realization of the elevation model elevation useful for the realization of an orthophoto, and finally the predisposition of the solid-orthophoto.

\section{THE AERIAL PLATFORM}

The system consists of a low cost vertical take-off and landing (Vertical Take Off and Landing) airplane called HexaKopter produced by Mikrokopter, sold as a kit to be assembled. As the name suggests, the aircraft is equipped with six rotating wings, weighs approximately $1.2 \mathrm{~kg}$ including batteries, and with a maximum load (payload) of $1 \mathrm{~kg}$. The complete kit provides some control cards and a set of navigation sensors as indicated in the diagram shown in Figure 1.

In particular, the following components are included:

- 6 Brushless motors (BL) equipped with 10" long propellers

- 6 cards Brushless Controls (BCs) that regulate the speed of rotation of the motors;

- 1 card Flight Control (FC) which uses a dedicated processor to receive commands sent by the operator on the ground via a radio control and to interpret them for the execution of the flight by defining the characteristics of the navigation of the aircraft on the basis of many sensors which are installed (three-axial MEM gyroscope by Analog Devices, three-axial accelerometer by STMicroelectonics, barometric measure based altimeter, and a digital compass) and adjusting, according to that information, the rotation speed of the engines (by using the $\mathrm{BCs}$ );

- 1 Radio control which allows driving the aircraft by a trained operator by the activation of flight modalities available in real time: setting/locking of the flight altitude defined by the altimeter, in flight locked position (hold), return to home, navigating to predetermined points (waypoints), and the use of the manual radio control through the carefree mode;

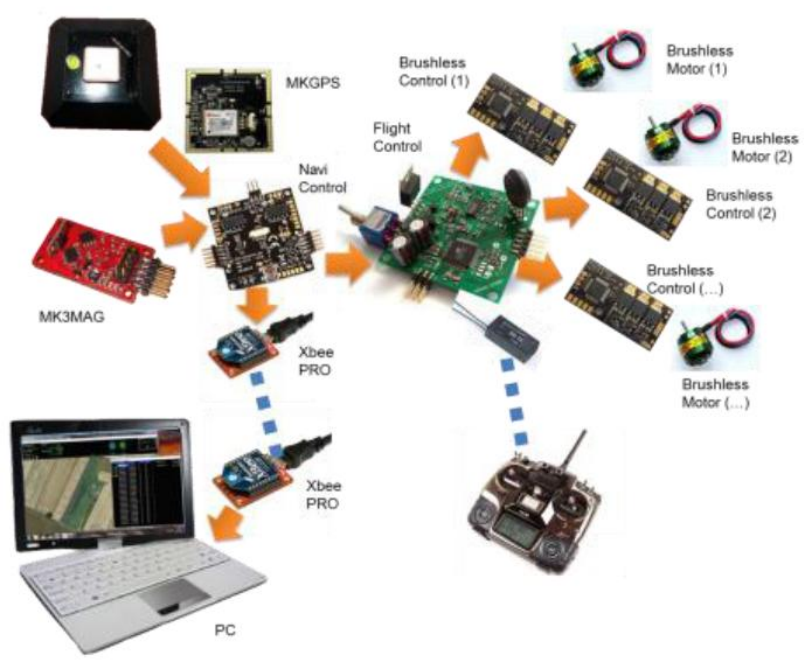

Figure 1. Schematic diagram of the system used based on Hexakopter by Mikrokopter

- 1 Ship Control (NC) that controls another dedicated processor and enables the required automatic controls (parking in a fixed position, back home, azimuthal rotation of the aircraft, navigation on waypoints) processing data acquired by the appropriate sensors of navigation, positioning (a GPS receiver) and attitude (Mk3MAG). In addition to allowing the real-time transmission of trajectory data and other parameters of telemetry, the NC stores them on a micro SD card which can be inserted in a suitable support;

- 1 GPS receiver (MKGPS) equipped with a small antenna integrated in the board. It comprises the sensor LEA 4H (or LEA 6S in the most recent versions) produced by Ublox with 
the possibility of DGPS positioning (EGNOS, WAAS, ...) for an accurate positioning with sub-metric accuracy. This sensor transmits, by serial transmission, the location information to the NC;

- 1 MK3MAG sensor equipped with a three-axial magnetometer that allows defining the attitude of the aircraft by the accurate measurement of the Earth's magnetic field: for a correct use of this sensor a calibration procedure is necessary to correctly define the initial status;

- 1 XBee Pro 2 based instrument connected by a wireless bidirectional serial connection with nominal flow rate of $1 \mathrm{~km}$ in open spaces connected to the serial port of the $\mathrm{NC}$ and to the $\mathrm{PC}$ used for the management of the operations during the execution of the flight;

- 1 PC that acts as the control station and allows performing all the necessary adjustments for a correct configuration of the system, defining waypoints and transmitting them to the local memories of the hexa-copter, and checking the telemetry transmitted during the flight to verify its effectiveness and to prevent any problems.
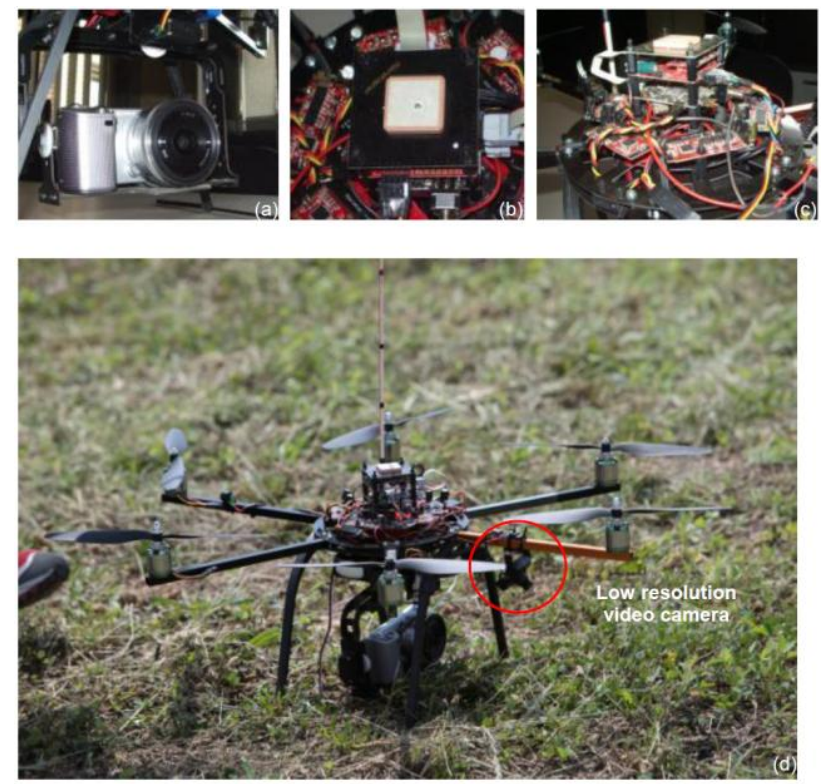

Figure 2. The digital camera Sony NEX-5 (a), The Ublox GPS receiver antenna (b), the electronic control unit (c). In (d) from the highest to the lowest part: BL, FC, NC, MK3Mag, MKGPS, and the Hexakopter by Mikrokopter

Figure 1 shows the system and highlights some of its details, included the digital camera used for the photogrammetric takes and video recording. In this application the Sony Nex_5 camera equipped with a CMOS sensor format of $23.5 \times 15.6 \mathrm{~mm}$ (APS$\mathrm{C}$ size) has been used. The pixel size is equal to 5 ( $\mathrm{M}$ for a total of 14 Megapixels, and pancakes optics of $16 \mathrm{~mm}$ focal length has been used. During the flight, shooting can be remotely controlled according to three different modes: two of them are controlled manually by an operator equipped with a radio control (single shot or timed sequence controlled by the FC). The third mode is automatic and runs with snap action on a certain event, defined during the planning phase of the flight (e.g., the path on waypoints governed by NC).

The digital camera is mounted on a servo-assisted support that allows electronically controlled rotations along 2 directions (around the $\mathrm{x}$ and $\mathrm{h}$-axis by arranging the camera in a nadir position). The movements of the support (and therefore the camera) are manually adjustable by means of the appropriate control on the radio control or automatically (by the FC) by compensating the rotations of the camera during the flight so that the optical axis always has almost the same direction (for example, always vertical). The third possible rotation around the $\zeta$ axis can be made by rotating the aircraft horizontally, either manually using the special control of the radio control (yaw), or using the automatic setting by fixing for each waypoint the needed azimuth of the aircraft.

To complete the system, a video camera at low resolution was installed (see Figure 2 (d)) with analog transmission in real time at a $2.4 \mathrm{MHz}$ frequency. This signal is received on the ground, converted by means of suitable USB instruments, and stored by the control station in video format (AVI).

\section{THE NEW MONITORING STRATEGY}

\subsection{The test site}

Thanks to the cooperation between the Politecnico di Torino and the University of Trieste in the metric documentation of the archaeological excavation of via Gemina in Aquileia (UD) (directed by Prof. Federica Fontana), it was possible to perform a test on Domus "dei putti danzanti". This name comes from the subject of a refined mosaic which adorned the boardroom. The Domus, partially excavated with systematic interventions from 2005 , is of late-ancient age, dating to the IV century A.D. Its strategic position (between the forum and the river port), its size, and its construction features, suggest that probably it belonged to a prominent personality of the time, perhaps an imperial official (Fontana, 2010; Murgia, 2010).

On the area, for some years, operations of integrated survey for the documentation and representation of the archaeological excavation have been organized. The purpose of these operations are directed, beyond the experimentation of instrumentation and non-conventional methods for archaeological survey, towards the realization of threedimensional databases from which to be able to derive either two-dimensional drawings (e.g., plans and vertical sections that are always an indispensable instrument of work) and 3D continuous surface models that allow rapid and effective views of the archeological sites, and also provide the possibility of indepth dimensional and interpretative analysis, thanks to the integration of photographic textures whose metric quality is controlled by the photogrammetric origin of the radiometric data [Spanò, 2011].

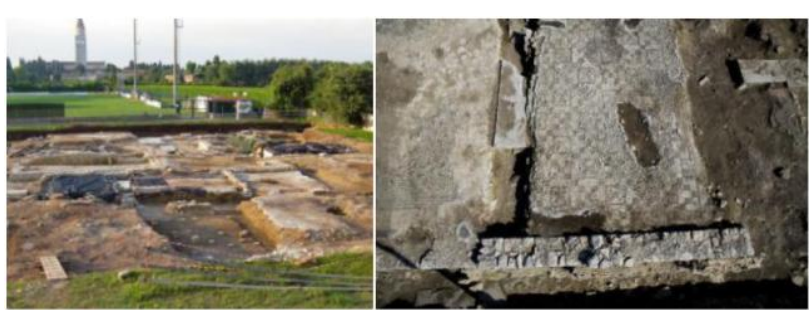

Figure 3. The Domus "dei putti danzanti"

\subsection{The data acquisition}

The acquisition of photogrammetric stereoscopic images was planned using an orthophoto of the area derived from the satellite Quickbird image (Figure 4a); two flights at different heights was planned. The choices of the geometry of the flight were carried out in a conservative way by considering the 
characteristics of the navigational sensors of the hexa-copter. The lowest flight develops itself over the remains of the roman road (cardo), located to the East of the excavation area, with an average height of flight of $10 \mathrm{~m}$, which provides a ground coverage of about $10 \times 15 \mathrm{~m} 2$. The forward overlap was set at $75 \%$, ensuring a base of about $4 \mathrm{~m}$, while the side overlap was set at $30 \%$ to avoid any stereoscopic hole due to tracking errors of the defined trajectories. A total of 36 frames was planned.

The highest flight covers the whole excavation area with an average height of flight of $18 \mathrm{~m}$, which provides a ground coverage of about $18 \times 27 \mathrm{~m} 2$. In this case the forward overlap was set at $75 \%$, ensuring a base of about $7.50 \mathrm{~m}$, while the side overlap was fixed at $40 \%$ so as to relieve, with the flight altitude provided, a greater possibility of instability of the aircraft relative to the planned trajectories. A total of 60 frames was planned for the highest flight.

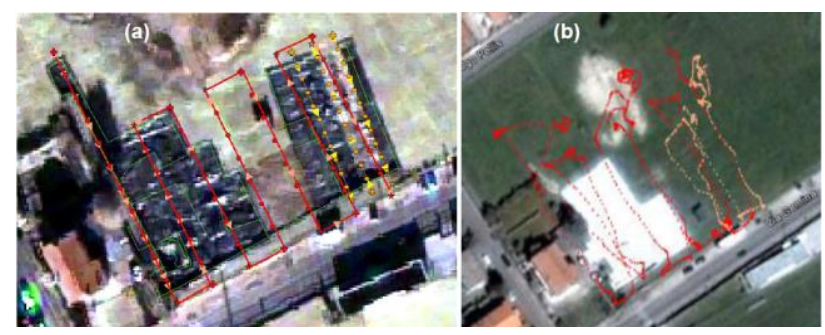

Figure 4. The flight planning on the Quickbird orthophoto (a) and the real trajectories followed by the aircraft (b): red lines described the highest flight and orange lines the lowest flight

From the plan of the flight, the coordinates of the start and end points of each strip have been extracted (indicated by, respectively, a cross and a star in Figure 4 (a)) and subsequently inserted into the control software of the ground station and sent to the local memory of the hexa-copter by using the Xbee wireless connection (see Figure 5).

The take-off was driven manually by the operator: when the aircraft reach the needed height, the operator ran the automatic flight control. The hexa-copter then flies towards the first waypoint (previously recorded on the control unit) and starts to run the planned strips.

Also the landing phase was carried out manually. Figure $4 \mathrm{~b}$ shows the trajectories actually followed by the aircraft (the highest flight in red, the lowest flight in orange), which highlights the difficulties in the pursuit of planned trajectories that have occurred due to the presence of wind gusts during the execution of the flight.
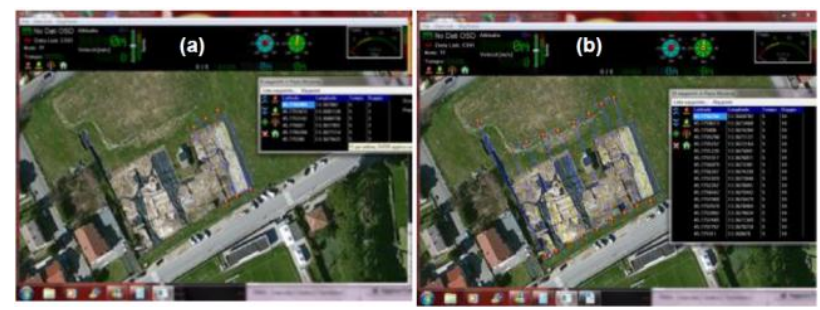

Figure 5. The planned flights input inside the control unit: lowest flight (a) and highest flight (b)

Before the execution of the flights, to be able to correctly perform the aerial triangulation and to obtain some feedback on the accuracy of the data acquired, a series of targets for use both as control points (GCPs - Ground Control Points) as well as check points (CPs) were positioned and measured over the whole area of the Domus. The measurements were performed with the use of a Total Station by referring the final coordinates of those points inside the coordinate system materialized on the area of the excavation of Via Gemina to guarantee effective monitoring of the excavation activities.

The GCPs were placed in order to satisfy the well-known requirements for performing a reliable aerial triangulation (i.e., following the OEEPE standards). The CPs were regularly distributed inside the whole excavation area in order to guarantee a reliable test of the accuracy of the oriented photogrammetric block.

\subsection{Photogrammetric block orientation}

The goal in this first step of processing the photogrammetric data was to follow a traditional procedure of aerial triangulation for the generation of an orthophoto of the entire excavation area of the Domus "dei putti danzanti" using images acquired from the highest flight. The images used have no regular overlaps and have different tilts due to the deleterious influence of the gusts of wind arising during the flight.

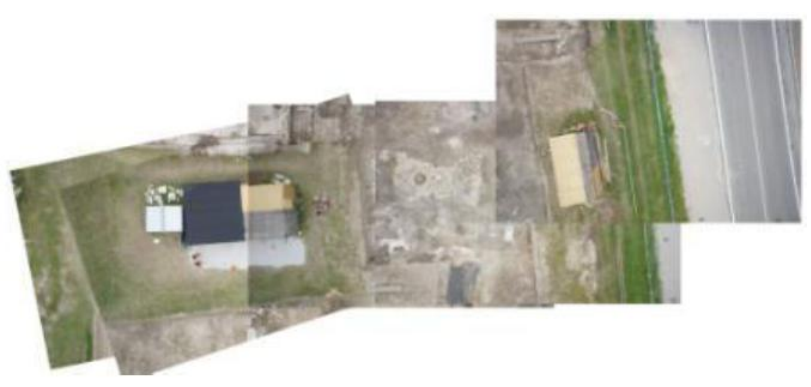

Figure 6. Set of images of the photogrammetric block acquired on the Domus.

Given the limited weight of a hexa-copter, these phenomena are considered to be a "normal case" under real operating conditions. However, thanks to the extreme flexibility of the aerial triangulation algorithm implemented in the photogrammetric software used for the data processing (LPSLeica Photogrammetric Suite), it was possible to successfully orient the 62 images acquired.

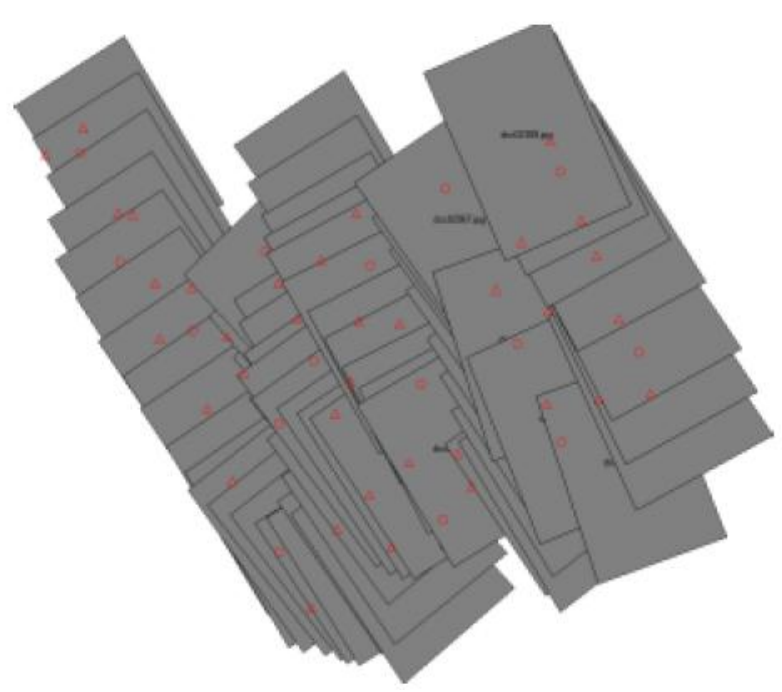

Figure 7. The oriented highest photogrammetric block 
For the orientation operations, 30 GCPs were used; furthermore, in order to improve the redundancy of the solving system, approximately 1200 tie points (TPs) were automatically identified by means of image matching. Figure 7 shows the graph of the triangulated photogrammetric block and Table 2 the precisions obtained on GCPs and the estimated accuracies on $15 \mathrm{CPs}$.

\begin{tabular}{|c|c|c|c|}
\hline Domus & $\sigma_{\mathrm{x}}[\mathrm{m}]$ & $\sigma_{\mathrm{y}}[\mathrm{m}]$ & $\sigma_{\mathrm{z}}[\mathrm{m}]$ \\
\hline GCPs (30) & 0.003 & 0.002 & 0.012 \\
\hline CPs (15) & 0.010 & 0.005 & 0.020 \\
\hline
\end{tabular}

Table 2. Estimated precision and accuracy of the highest photogrammetric block

\subsection{Final products}

By using the oriented photogrammetric block it was possible to survey the digital surface model (DSM) of the excavation site and then to generate the orthoprojection of all the images subsequently merged to form the orthophoto map of the whole archaeological site (see Figure 8).

By considering the accuracy obtained (see Table 2), it is possible to accept a nominal scale ranging from 1:200 up to 1:100 for the orthophoto produced.

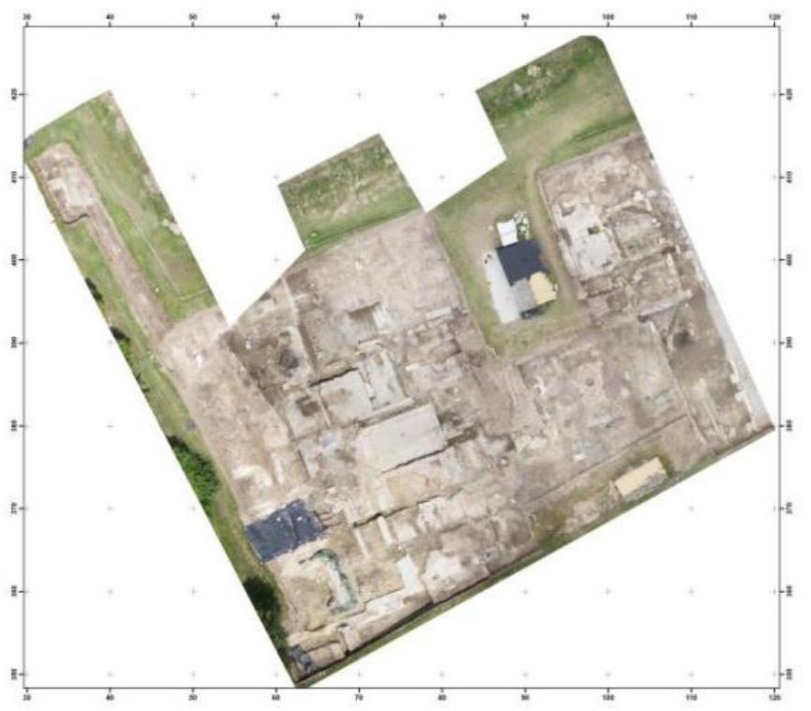

Figure 8. The orthophoto of the Domus "dei putti danzanti"

Another product that has been made, with both the twodimensional aspect typical of an orthophoto, and the height information typical of a DSM, is the solid orthophoto (OSP), [Dequal, 2003]. The OSP is a raster product which associates to each pixel in a geo-referenced matrix the chromatic information (and eventually radiometric multi-spectral information) and the height: in this way, the available data can be placed in their correct three-dimensional location even if the user can observe only in $2 \mathrm{D}$.

Given the intrinsic simplicity of raster data, an OSP can be viewed and interrogated by an user friendly viewer developed in the Visual Fortran environment using the graphical routines GINO v. 7.5 [Lingua, 2007]. The cartographic coordinates for each position of the cursor within the OSP are directly displayed, the user can use basic commands such as zooming and panning and can recover basic metric information such as distances, angles, areas, and volumes. In addition, it is possible to produce simple plans and sections of the described object. The software can be implemented on tablet PCs and other simple electronic tools, which is very useful when basic analyses have to be realized directly in the field.

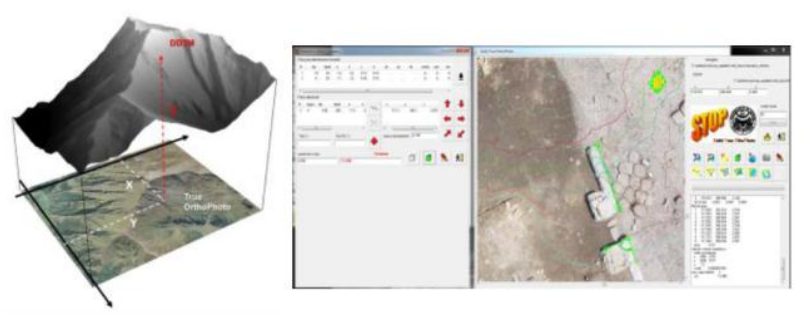

Figure 9. The SOP concept (left); the SOP (Solid OrthoPhoto) visualizer and the extraction of metric data (e.g., plans, sections, volume estimation, etc. — right)

\section{CONCLUSIONS}

Unconventional systems for photogrammetric image acquisition able to produce very large scale cartographic products allow reaching the necessary accuracies for the monitoring of an archaeological site during the excavation.

The present article showed that the low cost aerial system developed by the Research Group of Geomatics of the Politecnico di Torino can offer a practical and inexpensive solution to support archaeological analysis.

Given the time of realization of the flights (about 10 minutes for the highest flight) and the considerable automation of the data processing, this technique can be offered as a monitoring system for the work of daily advancement of an archaeological excavation, where the results of the excavations carried out during the day are the basis for the selection of the methods to be adopted in subsequent days.

Finally, the OSP, automatically produced after DSM extraction, allows the realization of the same graphic results (e.g. plans, sections, distance, angle and area measurements, etc.) usually obtained after a traditional Total Station survey.

The advantage of replacing the traditional approach by a modern photogrammetric system (UAV and OSP) is the possibility of storing the image information as a metric archive of the real situation of the excavated area during the working period from which, during the post-analysis of the obtained results, additional metric information, usually not necessary during the excavation, can be extracted.

\section{REFERENCES}

Aber, J. S., Aber, S. W., Pavri, F., 2002. Unmanned smallformat aerial photography from kites for acquiring large scale, high-resolution, multiview-angle imagery. ISPRS Archives, Pecora 15/Land Satellite Information IV/ISPRS Commission I/FIEOS, Denver, CO, USA.

Altan, M. O., Celikoyan, T. M., Kemper, G., Toz, G., 2004. Balloon photogrammetry for cultural heritage. ISPRS Archives Vol. XXXV-B5, XX ISPRS Congress, Istanbul, Turkey, pp. 964968.

Bendea, H., Boccardo, P., Dequal, S., Giulio Tonolo, F., Marenchino, D., Piras, M., 2008. Low cost UAV for postdisaster assessment. ISPRS Archives Vol. XXXVII, ISPRS Congress, Beijing, China, pp. 1373-1380. 
Bitelli, G., Tini, M. A., Vittuari, L., 2003. Low-height aerial photogrammetry for archaeological orthoimaging production. ISPRS Archives Vol. XXXIV, Part 5/W12, pp. 55-59.

Bogacki, M., Malkowski, W., Misiewicz, K., 2008. Kite aerial photography (KAP) as a tool for completing GIS models. Ptolemais (Libya) case study. Lasaponara, R., Masini, N., Aracne (Eds.), Remote Sensing for Archaeology and Cultural Heritage Management. Proceedings of the 1st International EARSeL Workshop, CNR, Roma, pp. 329-332.

Bornaz L., Dequal S., Lingua A., 2006. L'ortofoto solida di precisione: un prodotto innovativo per la rappresentazione e la gestione dei dati tridimensionali. Atti Conferenza nazionale SIFET, Taranto.

Bornaz, L., Dequal, S., 2003. The solid image: A new concept and its applications. ISPRS Archives, Vol. 34, Part 6/W12.

Celikoyan, T. M., Altan, M. O., Toz, G., 2003. Evaluation of a theatre by using low-altitude aerial and terrestrial photogrammetry. ISPRS Archives Vol. XXXIV-5/C15, CIPA, Antalya, Turkey, pp. 375-379. ISSN: 1682-1750.

Chiabrando, F., Costamagna, E., Spano', A.,Rinaudo, F., 2010. Very close nadiral images: a proposal for quick digging survey. ISPRS Archives, vol. XXXVIII/5, pp. 155-160. ISSN: 16821750.

Chiabrando, F., Nex, F., Piatti, D., Rinaudo, F., 2011. UAV and RPV systems for photogrammetric surveys in archaelogical areas: Two tests in the Piedmont region (Italy). Journal of Archaeological Science, Volume 38, Issue 3, March 2011, Pages 697-710.

Cunningham, K., Walker, G., Stahlke, E., Wilson R., 2011. Cadastral audit and assessments using unmanned aerial systems. ISPRS Archives, Vol. XXXVIII-1/C22 UAV-g 2011, Conference on Unmanned Aerial Vehicle in Geomatics, Zurich, Switzerland.

Dequal S., Lingua A., 2003. True orthophoto of the whole town of Turin. ISPRS Archives XXXIV-5/C15, pp. 263-268 ISSN: 1682-1750.

Eisenbeiss, H., Lambers, K., Sauerbier, M., Zhang, L., 2005. Photogrammetric documentation of an archaeological site (Palpa, Peru) using an autonomous model helicopter. ISPRS Archives Vol. XXXIV-5/C34, CIPA, Torino, Italy, pp. 238-243. ISSN: $1682-1750$.

Fontana F., 2010, La domus 'dei putti danzanti'. Forma urbis, Anno XV, n. 12, Dicembre 2010, pp. 7-9.

Fotinopoulos, V., 2004. Balloon photogrammetry for archaeological surveys. ISPRS Archives Vol. XXXV-B5, XX ISPRS Congress, Istanbul, Turkey, pp. 504-507.

Haala, N., Cramer, M., Weimer, F., Trittler, M., 2011. Performance test on UAV-based photogrammetric data collection. ISPRS Archives, Vol. XXXVIII-1/C22 UAV-g 2011, Conference on Unmanned Aerial Vehicle in Geomatics, Zurich, Switzerland.

International Unmanned Aerial System Community, 2008. Unmanned Aircraft System. The Global Perspective 2008/2009.

Lingua A., Agosto E., Del Bianco P., 2007. L'ortofoto solida di precisione a grandissima scala in ambito urbano. Atti XI conferenza nazionale Asita. Torino, Italy.

Murgia E., 2010, La domus 'dei putti danzanti'. Archeologia Viva, anno XXIX, n. 141, maggio/giugno: 21.

Patias, P., Saatsoglou-Paliadeli, C., Georgoula, O., Pateraki, M., Stamnas, A., Kyriakou, N., 2007. Photogrammetric documentation and digital representation of the Macedonian palace in Vergina-Aegeae. ISPRS Archives Vol. XXXVI-5/C53, XXI CIPA Symposium, Athens, Greece.

Patias P., Olga G., Charalampos G., Anastasios G., Tassopoulou M., 2009. Photogrammetric documentation and digital representation of excavations at Keros island in the Cyclades. ISPRS Archives Vol. XXXVIII-3/W8, XXII CIPA Symposium, Kyoto, Japan.

Remondino, F., Gruen, A., Von Schwerin, J., Eisenbeiss, H., Rizzi, A., Sauerbier, M., Richards-Rissetto, H., 2009: Multisensors 3D documentation of the Maya site of Copan. ISPRS Archives Vol. XXXVIII-3/W8, XXII CIPA Symposium, Kyoto, Japan.

Remondino, F., Barazzetti, L., Nex, F., Scaioni, M., Sarazzi, D., 2011. UAV photogrammetry for mapping and $3 \mathrm{~d}$ modeling Current status and future perspectives. ISPRS Archives, Vol. XXXVIII-1/C22 UAV-g 2011, Conference on Unmanned Aerial Vehicle in Geomatics, Zurich, Switzerland.

Scaioni M., Barazzetti L., Brumana R., Cuca B., Fassi F., Prandi F., 2009. RC-Heli and structure \& motion techniques for the 3-D reconstruction of a Milan dome spire. IAPRSSIS, Vol. 38, Part 5/W1, Trento, Italy (on CDROM).

Scholtz, A., Kaschwich, C., Krüger, A., Kufieta, K., Schnetter, P., Wilkens, C.-S., Krüger T., Vörsmann, P., 2011. Development of a new multi-purpose uas for scientific application. ISPRS Archives, Vol. XXXVIII-1/C22 UAV-g 2011, Conference on Unmanned Aerial Vehicle in Geomatics, Zurich, Switzerland.

Spanò, A., Chiabrando, F., Rinaudo, F., 2011, Contributi della geomatica ai temi delle ricerche archeologiche di Aquileia, in L'edilizia privata ad Aquileia e nel suo territorio, Atti delle giornate di studio, Padova 21-22 febbraio 2011.

Spatalas, S., Tsioukas, V., Daniil, M., 2006. The use of remote controlled helicopter for the recording of large scale urban and suburban sites. In: Proceedings of the Scientific Conference. Culture of Representation, Xanthi, Greece.

Skarlatos, D., Theodoridou, S., Glabenas, D., 2004. Archaeological surveys in Greece using radiocontrolled helicopter. In: Proceedings of the FIG Working Week 2004, International Athenaeum Athens, Athens, Greece.

Theodoridou, S., Tokmakidis, K., Skarlatos, D., 2000. Use of radio-controlled model helicopters in archaeology surveying and in building construction industry. ISPRS Archives Vol. XXXIII-B5, XIX ISPRS Congress, Amsterdam, The Netherlands.

Tokmakidis, K., Skarlatos, D., 2000. Mapping excavations and archaeological sites using close range photos. In: Proceedings of the ISPRS Commission V Symposium, WG V/4, Corfu, Greece.

Verhoeven, G. J. J., 2009. Providing an archaeological bird'seye view-An overall picture of ground-based means to execute low-altitude aerial photography (LAAP) in archaeology. Archaeological Prospection 16(4), 233-249.

\section{AKNOWLEDGEMENTS}

Prof. Federica Fontana (Archeological site Director) and all the students of the Archeological Summer School of the Università di Trieste for their warm hospitality and rich collaboration. 